\title{
Application and efficacy of extracorporeal shockwave treatment for knee osteoarthritis: A systematic review and meta-analysis
}

\author{
TENGQI LI ${ }^{1,2}$, JINHUI MA ${ }^{1}$, TINGTING ZHAO ${ }^{1}$, FUQIANG GAO ${ }^{1}$ and WEI SUN ${ }^{1}$ \\ ${ }^{1}$ Department of Orthopedic Surgery, China-Japan Friendship Hospital; ${ }^{2}$ Department of Orthopedic Surgery, \\ Peking University China-Japan Friendship School of Clinical Medicine, Beijing 100029, P.R. China
}

Received November 24, 2018; Accepted July 5, 2019

DOI: 10.3892/etm.2019.7897

\begin{abstract}
The increasing aging of the world population is accompanied by a rise in the incidence of knee osteoarthritis (KOA). There has been a growing interest in shockwave treatment for orthopedic diseases, including KOA. In previous trials, extracorporeal shockwave therapy (ESWT) was compared to physical therapy or placebo in the treatment of KOA. However, the efficacy and safety of ESWT for KOA remains disputed. The present meta-analysis assessed the effects of ESWT in KOA. The PubMed, Medline, Embase, Web of Science, Research Gate and the Cochrane Library were searched to identify comparative studies involving ESWT for patients with KOA. The outcome indicators included the visual analog scale (VAS) score, range of motion (ROM), the Lequesne index (LI) and the Western Ontario and McMaster Universities osteoarthritis index (WOMAC). In the comparison of the ESWT vs. placebo groups, the primary outcomes included the VAS score and ROM, while the LI was the secondary outcome. In the comparison of the ESWT vs. physical therapy groups, the primary outcomes included the pain score and ROM, while the secondary outcome was the WOMAC index. Relevant data were analyzed using RevMan v5.3. The ESWT group had a lower VAS core, larger ROM and a better LI than the placebo group after 1 month of therapy $(\mathrm{P}<0.05)$. Furthermore, at 1 month post-therapy, the ESWT group had a lower VAS score, larger ROM and
\end{abstract}

Correspondence to: Dr Wei Sun or Dr Fuqiang Gao, Department of Orthopedic Surgery, China-Japan Friendship Hospital, 2 Yinghua East Street, Chaoyang, Beijing 100029, P.R. China

E-mail: doctor_sunwei@126.com

E-mail: gaofuqiang@bjmu.edu.cn

Abbreviations: ESWT, extracorporeal shockwave therapy; OA, osteoarthritis; KOA, knee OA; ROM, range of motion; VAS, visual analogue score; LI, Lequesne index; WOMAC, Western Ontario and McMaster Universities osteoarthritis index; RCT, randomized controlled trial; MD, mean difference; CI, confidence interval; SD, standard deviation

Key words: shockwave, osteoarthritis, knee, physical therapy, meta-analysis a better WOMAC than the physical therapy group $(\mathrm{P}<0.05)$. The outcomes regarding pain, ROM, LI and WOMAC were significantly different between the two different groups $(\mathrm{P}<0.05)$. The present meta-analysis suggested that ESWT may achieve a better therapeutic effect for patients with KOA as compared to physical therapy. However, high-quality trials with large sample sizes are essential to substantiate these results.

\section{Introduction}

The increase in the aging population has led to a rise in the incidence of osteoarthritis (OA), which is accompanied by a high economic burden on the whole society. OA is the most common type of degenerative arthritis among individuals from different countries; also, since it progresses slowly, it is not detected and diagnosed at the early stage, causing a delay of treatment and a limitation of its efficacy (1). OA frequently occurs in active joints, including the knee and hip. According to epidemiological studies, the incidence of individuals $>55$ years of age suffering from OA is $44-70 \%$, and $10 \%$ of patients experience pain in the joints, along with reduced muscle strength and decline in joint stability and mobility, which in turn results in walking difficulties and severely affects daily life (2-4). Typically, OA affects large weight-bearing joints, particularly the knees, where the disease is disabling, as the knees participate in several basic activities, including standing up from a seated position, climbing, walking and running (5). Knee OA (KOA) has various clinical symptoms, including pain associated with muscles and tendons, which may decrease the range of motion, crepitus with movement and joint effusion in the knee. Reportedly, the incidence of KOA among individuals $>60$ years of age is $50 \%$ and that of individuals aged $>75$ years is $\sim 80 \%$ (6).

In recent years, there has been a growing interest in shockwave treatment for orthopedic diseases, including KOA. Several musculoskeletal diseases have been treated with shockwave therapy (7). The effect of the therapy includes pain relief, improvement of joint movement and prevention of the progression of avascular necrosis $(8,9)$. Shockwave treatment has several advantages, including non-invasiveness, low complication rate, no hospitalization and low cost as compared to other approaches. Accordingly, 
shockwave treatment is beneficial at the specific stage when surgery was selected as the approach for several associated diseases $(10,11)$. Extracorporeal shockwave therapy (ESWT) has been increasingly used in patients suffering from KOA (12-14), and in clinical trials, ESWT was compared with physical therapy or placebo. The outcome indicators included visual analog scale (VAS) score, range of motion (ROM), Lequesne index (LI) and Western Ontario and McMaster Universities osteoarthritis index (WOMAC). A majority of the studies indicated that ESWT offered better pain relief than placebo. Conversely, the study by Imamura et al (15) did not demonstrate any statistically significant difference between ESWT and placebo treatment in pain control on patients with severe KOA and disabling pain. In addition, the results regarding other outcome measures were different among various studies. Thus, the present meta-analysis was performed to ascertain whether ESWT is superior to physical or placebo therapy with regard to all outcome indicators.

\section{Materials and methods}

Search strategy. The present meta-analysis was in accordance with the Preferred Reporting Items for Systematic Reviews and Meta-Analyses guidelines for the meta-analysis of intervention trials (16).

The PubMed, Medline, Embase, Web of Science, Research Gate and the Cochrane Library were searched for comparative studies involving ESWT for patients with KOA published until February 2019. The search terms were as follows: 'Extracorporeal shockwave therapy' OR 'physical therapy' OR 'exercise therapy' AND 'osteoarthritis' OR 'knee osteoarthritis' OR 'knee arthritis'. The language of the publications was limited to English. The title and abstract of the studies identified in the search were reviewed to exclude irrelevant studies. The reference lists of all eligible studies and relevant reviews were searched manually for additional trials.

Inclusion criteria and study selection. First, the studies comparing ESWT to physical therapy or ESWT to placebo in patients suffering from KOA were identified. The primary outcomes included the pain score and ROM, while the secondary outcomes were the LI and WOMAC. Subsequently, studies comparing ESWT and physical therapy in patients with KOA were selected, in which the primary outcomes included the pain score and ROM, while the secondary outcome was the WOMAC. Studies that reported on at least one outcome measure were included, and those without the outcome measures of interest were excluded. Furthermore, studies that did not contain data that could be used for analysis were excluded. Letters, comments, editorials and practice guidelines were also excluded.

Data extraction and quality assessment. The authors read all titles and abstracts that were identified according to the eligibility criteria. The articles meeting the inclusion criteria were reviewed in further detail. Further discussion amongst the listed authors settled any differences in order to reach a consensus. The authors used standardized tables to extract data on patient characteristics (age, sex, and so on), interventions, and outcomes in duplicate. Other formats data [i.e., median, interquartile range] were converted to mean \pm standard deviation according to the Cochrane Handbook (17). If there was no report in digital format then data was extracted from the figures published by manual measurement. If necessary, we contacted the corresponding author to confirm the information fulfilled the criteria described above.

The risk of bias in each study was assessed. We evaluated the rigor of the randomized process, the concealment of treatment allocations, the process of blinding, data integrity and the results according the Cochrane Collaboration Recommendations (17). For the purpose of this meta-analysis, randomization, concealment of treatment allocation, and blinding and integrity were identified as key areas to determine the risk of bias. In each study included, the risk of bias was determined to be low when all key areas were fully assessed. When one or more key areas are not clearly described, the risk of bias is identified as unclear. When one or more key areas are inadequate, the risk of bias is determined to be high. The quality of evidence for each outcome was determined by considering the risk of bias. The assessment of the risk of bias is shown in Fig. 1.

All calculations were made using the mean difference (MD) with a 95\% CI that was calculated for continuous data using RevMan v5.3 (16). The heterogeneity among studies was estimated using $\mathrm{I}^{2}$ statistics and substantial heterogeneity was considered if $\mathrm{I}^{2}>50 \%$. The random-effects model was adopted for the meta-analysis of all data. Because the random effect model could be used in all cases, if the heterogeneity was small, then there would be no large difference between random effect model and fixed effect model. When the heterogeneity is large, only the random effect model could be used therefore the random effect model was used in all cases. $\mathrm{P}<0.05$ was considered to indicate statistical significance. Sensitivity analysis explored the impact of individual studies by removing one study at a time in sequence.

\section{Results}

Search results. Initially, a total of 127 studies were retrieved, among which 47 were identified as duplicates and were excluded. The titles and abstracts of the remaining studies were read and 20 studies that did not fulfill the inclusion criteria were excluded. Subsequently, 24 articles that were not randomized controlled trials (RCTs) were also excluded (Fig. 2). Finally, seven studies comprising 366 patients were included for data extraction and meta-analysis. Of these, 169 patients were in the ESWT group $(15,18-23)$ and 140 in the placebo group $(15,18-21)$ and 57 patients belonged to the physical therapy group $(18,22,23)$.

Study characteristics and quality assessment. The eligible studies included were published between 2013 and 2017. All of the studies were RCTs. The subjects in the two groups had similar demographic data. All trials used ESWT, except for the study by Imamura et al (15), which used radial ESWT. This may have led to heterogeneity in data analysis. In the ESWT group, all patients underwent treatment at least once per week and the total number of sessions exceeded 3 times. In the placebo group, the patients received the same number 


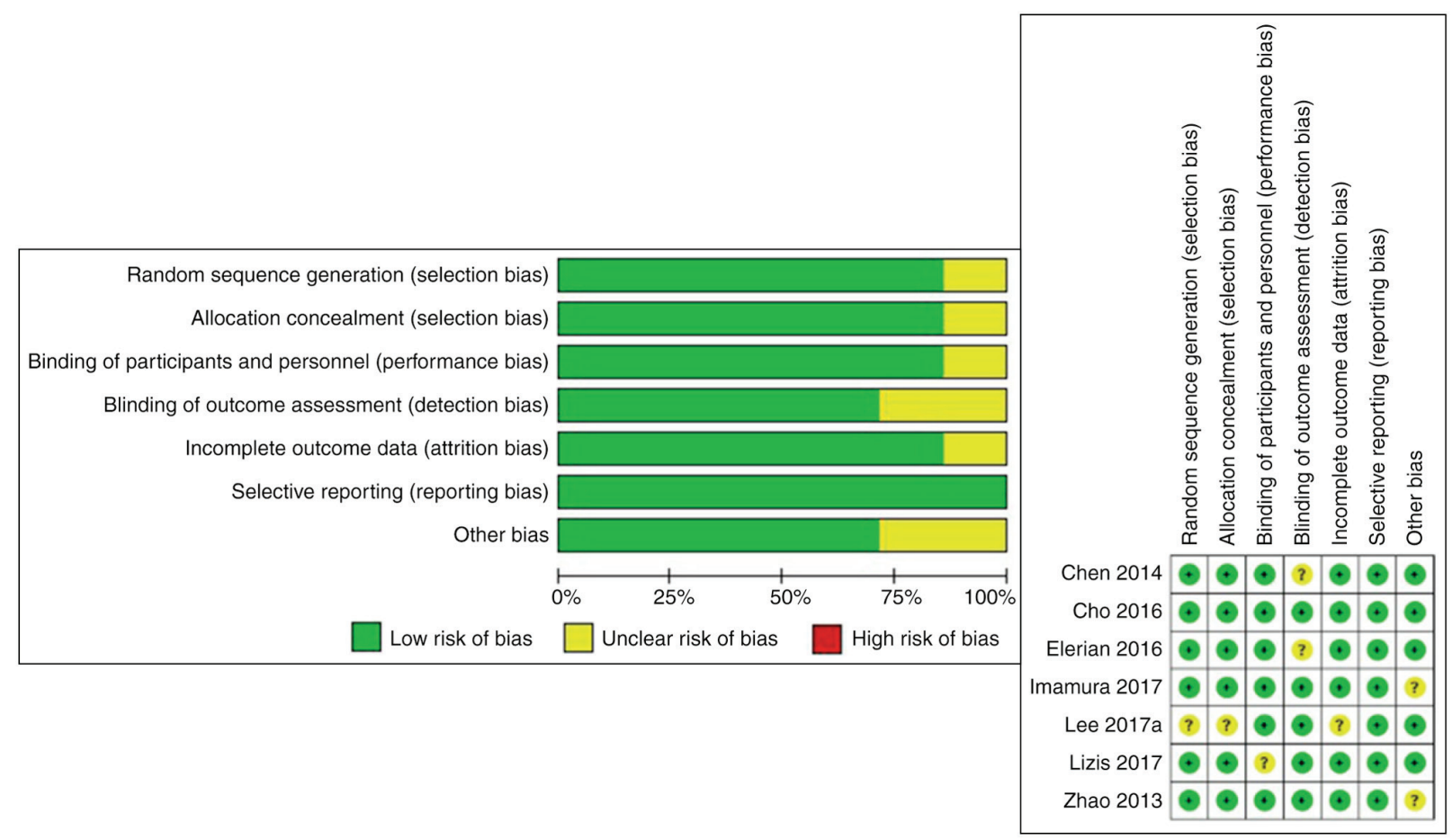

Figure 1. Summary of risk of bias.

of impulses of sham stimulation with an energy of 0 on the same area so that they did not realize they were not treated. In the physical therapy group, participants performed various exercises, including stretching, global flexion-extension of the lower limb and strengthening. However, not all studies assessed the same indicators. In the present meta-analysis, the outcomes of muscle peak torques and the modified Barthel index were not analyzed owing to insufficient data.

Meta-analysis of ESWT vs. placebo. A total of four studies $(15,18-20)$ involving 218 patients reported on the VAS score around 4 weeks following completion of therapy. The statistical heterogeneity between the studies was high as evaluated by a random-effects model $\left(\mathrm{P}=0.06, \mathrm{I}^{2}=59 \%\right.$; Fig. 3). However, after the fourth study was removed, the statistical heterogeneity of the remaining three studies was significantly reduced $\left(\mathrm{P}=0.33, \mathrm{I}^{2}=9 \%\right.$; Fig. 4). Meta-analysis of the data revealed that the VAS scores of ESTW patients were significantly lower than those in the placebo-treated patients after therapy after 1 month $(\mathrm{MD}=-2.35,95 \% \mathrm{CI}$ : -2.92 to $-1.79, \mathrm{P}<0.00001)$.

A total of two studies $(18,20)$ including 95 patients reported on the ROM of knees after therapy for 1 month. The statistical heterogeneity between the studies was low as assessed by a random-effects model $\left(\mathrm{P}=0.20, \mathrm{I}^{2}=38 \%\right)$. The increase in the ROM scores in the ESWT group was significantly higher than that in the placebo group after treatment ( $\mathrm{MD}=17.58,95 \%$ CI: 12.88-22.28, $\mathrm{P}<0.00001$; Fig. 5).

The LI after treatment for 1 month was assessed by two studies $(18,21)$ comprising 116 patients. The statistical heterogeneity between the studies was reliable, as assessed by a random-effects model $\left(\mathrm{P}=0.27, \mathrm{I}^{2}=18 \%\right)$. The ESWT group scored significantly lower than the placebo group ( $\mathrm{MD}=-3.06,95 \% \mathrm{CI}:-3.90$ to $-2.21, \mathrm{P}<0.00001$; Fig. 6).

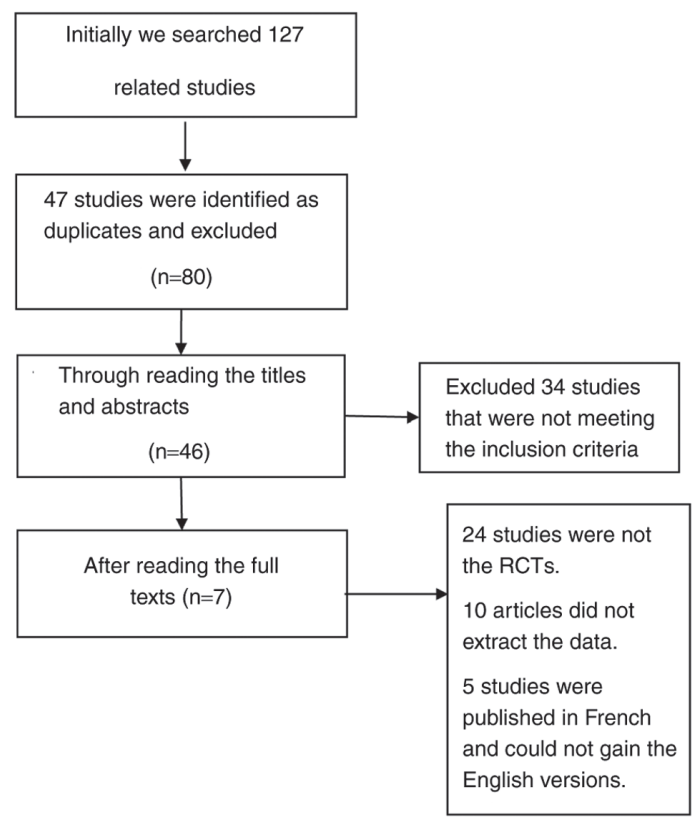

Figure 2. Flowchart of the study selection. RCT, randomized controlled trial.

Meta-analysis of ESWT vs. physical therapy. A total of two studies $(18,22)$ involving 75 patients reported on the pain score around 5 weeks following completion of therapy. The statistical heterogeneity between the studies was considerable according to the random-effects model $\left(\mathrm{P}=0.13, \mathrm{I}^{2}=56 \%\right)$. The VAS scores of the ESTW patients were significantly lower than of those with physical therapy for $\sim 1$ month $(\mathrm{MD}=-1.98$, 95\% CI: -2.93 to $-1.03, \mathrm{P}<0.00001$; Fig. 7 ).

The ROM of knees around 5 weeks following completion of therapy was reported by two studies $(18,23)$ including 95 


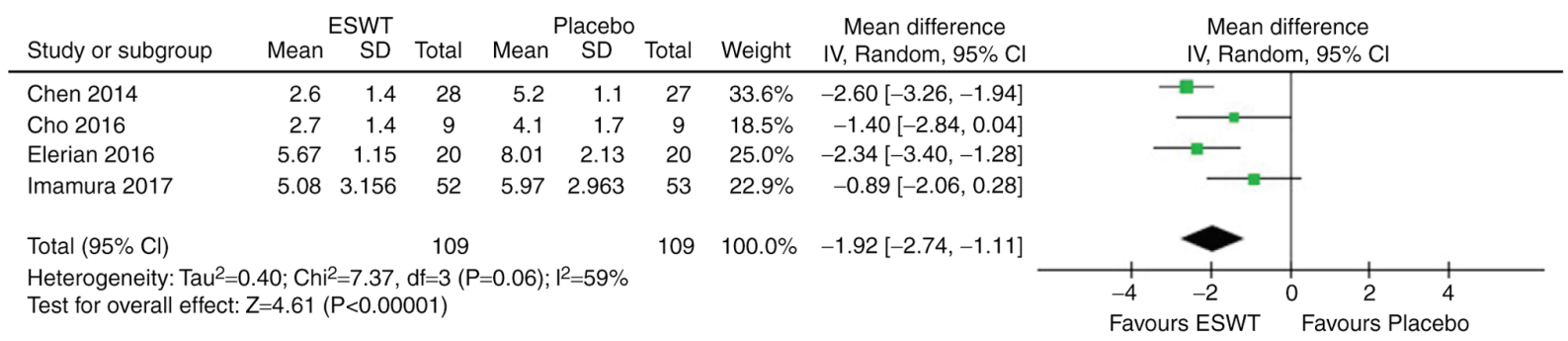

Figure 3. Forest plot for the visual analog scale score compared between the ESWT and placebo groups. ESWT, extracorporeal shockwave therapy; SD, standard deviation; IV, inverse variance; df, degrees of freedom.

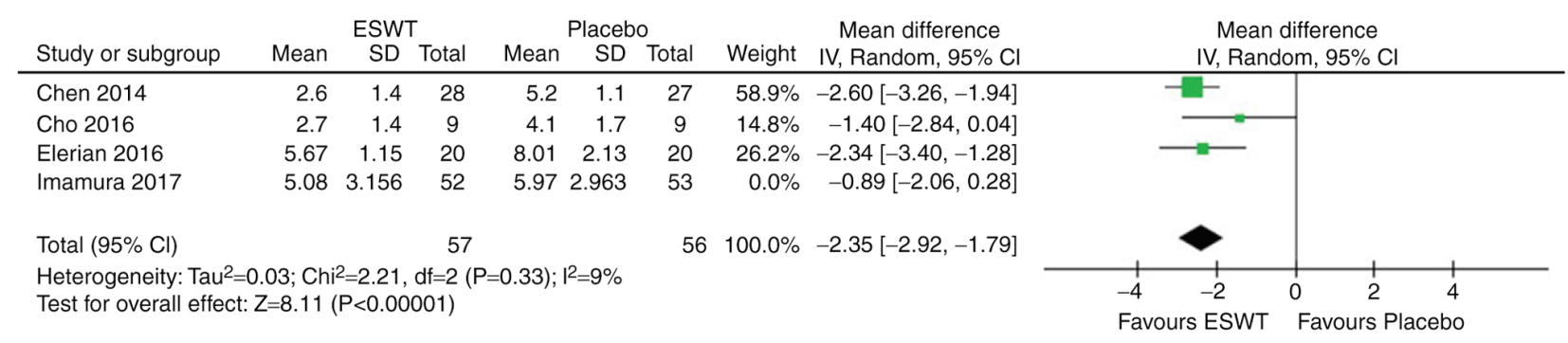

Figure 4. Sensitivity analysis after removal of the fourth study. ESWT, extracorporeal shockwave therapy; SD, standard deviation; IV, inverse variance; df, degrees of freedom.

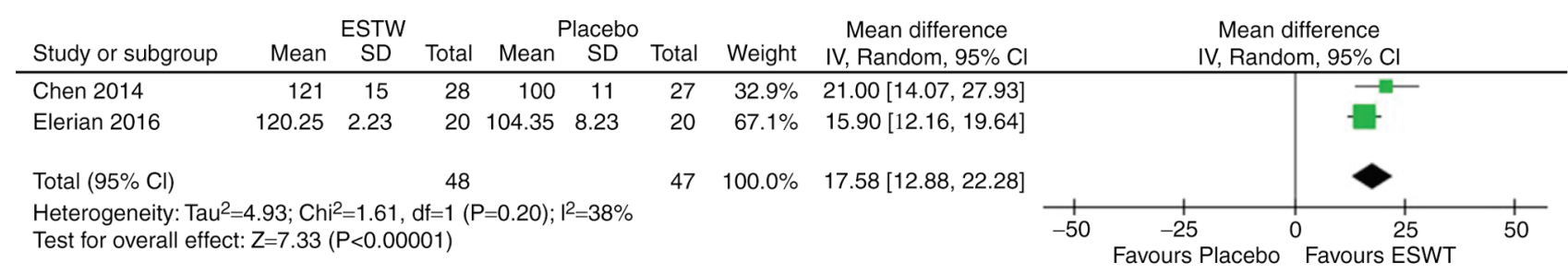

Figure 5. Forest plot for the range of motion compared between the ESWT and placebo groups. ESWT, extracorporeal shockwave therapy; SD, standard deviation; IV, inverse variance; df, degrees of freedom.

patients. The statistical heterogeneity between the studies was acceptable using a random-effects model $(\mathrm{P}=0.20$, $\mathrm{I}^{2}=39 \%$ ). The increase in the ROM scores during the treatment in the ESWT group was significantly higher than that in the physical therapy group (MD=11.69, 95\% CI: 6.40-16.98, $\mathrm{P}<0.00001$; Fig. 8).

Furthermore, two studies $(22,23)$ comprising 60 patients described the WOMAC around 4 weeks following completion of therapy. The statistical heterogeneity between the studies was low according to analysis with the random-effects model $\left(\mathrm{P}=0.77, \mathrm{I}^{2}=0 \%\right)$. The WOMAC in the ESWT patients exhibited a greater decrease than that in the physical therapy patients after treatment $(\mathrm{MD}=-15.38,95 \% \mathrm{CI}:-18.87$ to $-11.89, \mathrm{P}<0.00001$; Fig. 9).

\section{Discussion}

To the best of our knowledge, the present study was the first meta-analysis to investigate the efficacy and safety of ESWT as compared to physical therapy or placebo for the treatment of KOA. A series of RCTs on shockwave therapy has been performed for OA. In the ESWT group vs. placebo group analysis, the outcome indicators included were VAS, ROM and LI. Concerning pain control, one of the studies (15) indicated that shockwaves were not different from placebo in relieving severe pain for KOA. However, the combined analysis indicated that the shockwave group was significantly better than the placebo group, which was consistent with the individual studies. Furthermore, when the four studies were analyzed simultaneously, the heterogeneity was high $\left(\mathrm{P}=0.06, \mathrm{I}^{2}=59 \%\right)$. When the fourth study (15) was removed, the heterogeneity became reliable $\left(\mathrm{P}=0.33, \mathrm{I}^{2}=9 \%\right)$. Thus, it may be concluded that the heterogeneity of outcome indicators in this group originates from the last study (15). In addition, Imamura et al (15) used radial ESWT, which differs from shockwave equipment used in the other research studies. Furthermore, the patients selected were females and the pain was generally severe. The VAS score reached 7 points prior to the treatment whilst other studies reached around 5 points and this may have been the source of heterogeneity. Two studies $(18,20)$ assessed ROM and LI. Of note, the comprehensive analysis demonstrated that the patients in the shockwave group had a significantly greater ROM than the placebo group after treatment, and also, the LI was significantly reduced with acceptable heterogeneity, and hence, the result was reliable. However, the limited number of studies 


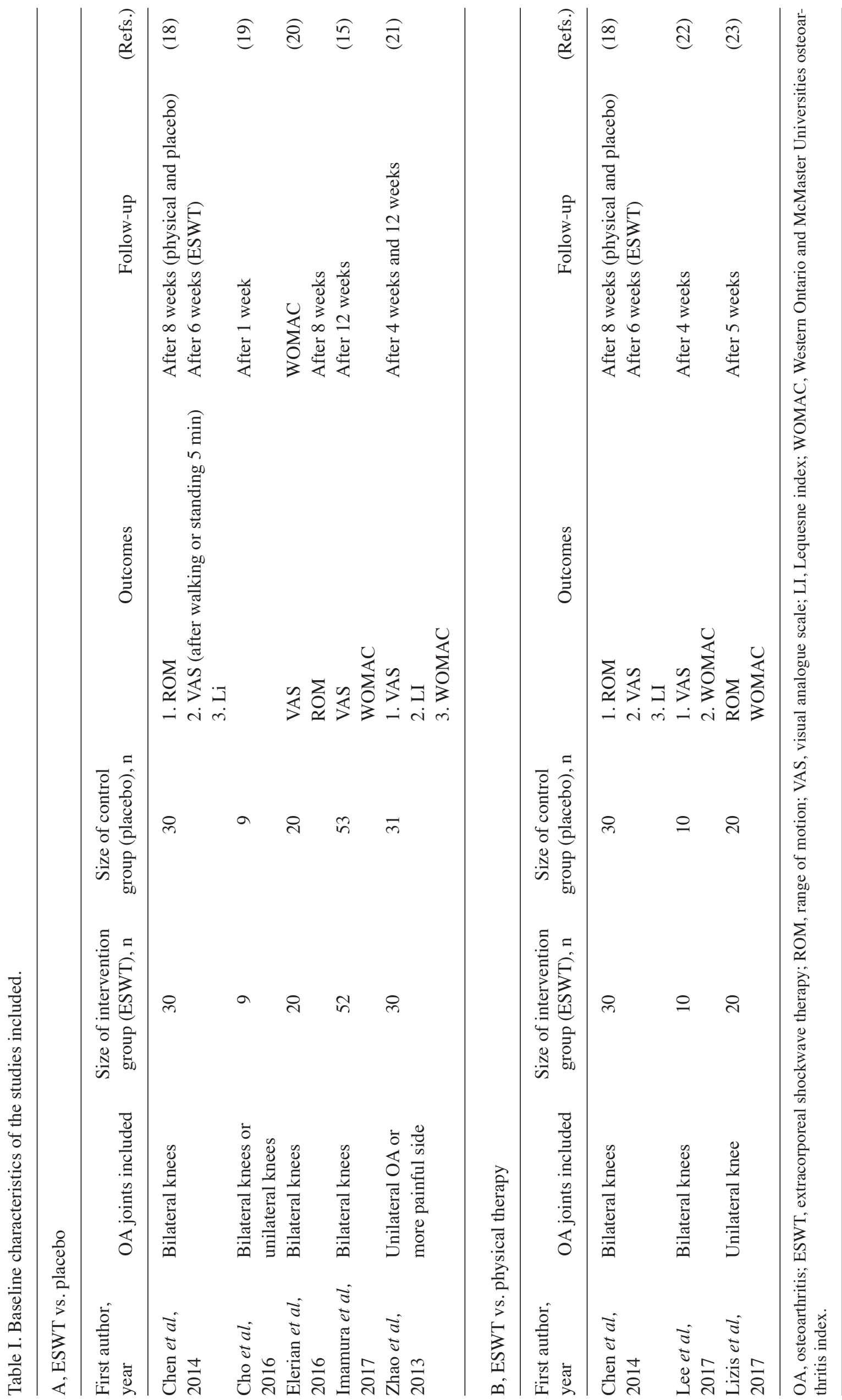




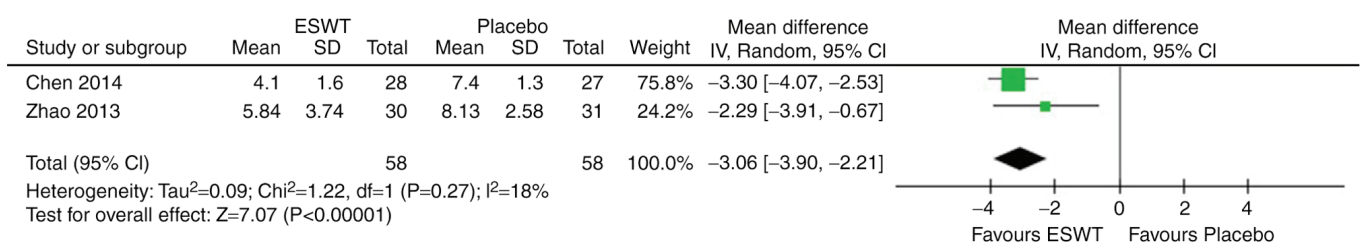

Figure 6. Forest plot for the Lequesne index compared between the ESWT and placebo groups. ESWT, extracorporeal shockwave therapy; SD, standard deviation; IV, inverse variance; df, degrees of freedom.

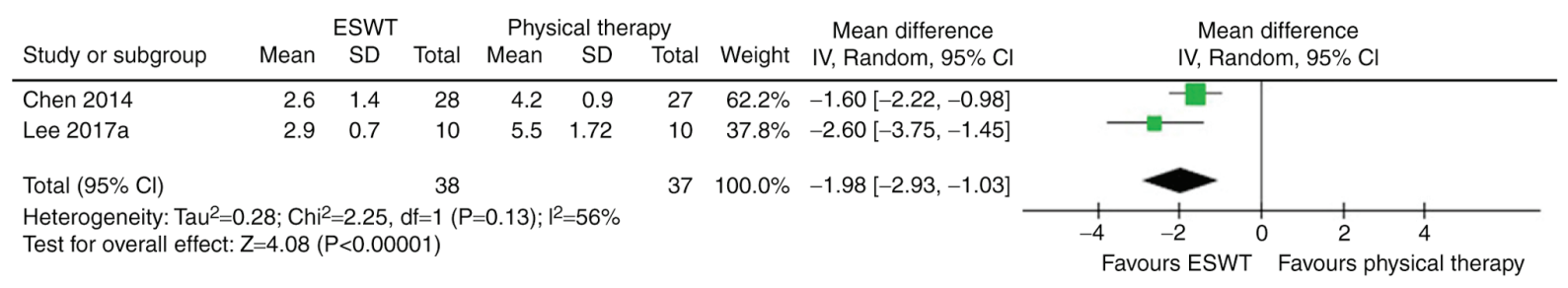

Figure 7. Forest plot for the visual analog scale score compared between the and physical therapy groups. ESWT, extracorporeal shockwave therapy; $\mathrm{SD}$, standard deviation; IV, inverse variance; df, degrees of freedom.

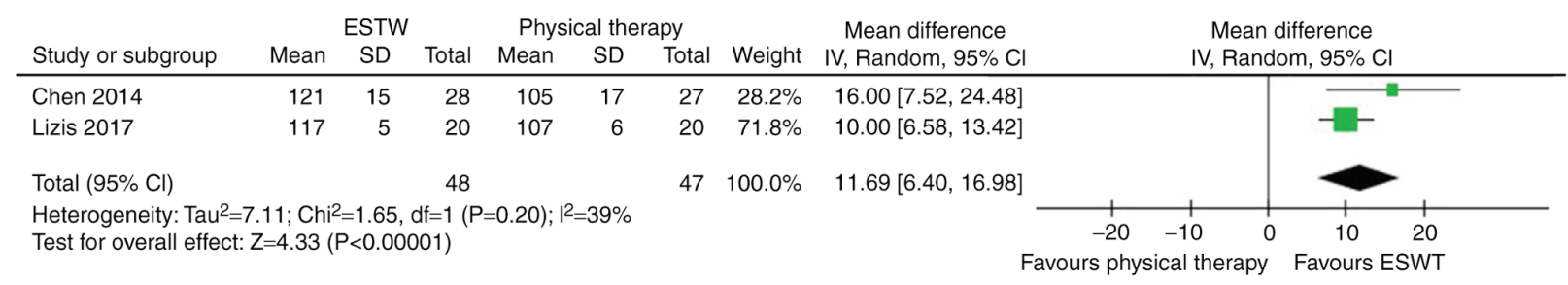

Figure 8. Forest plot for the range of motion compared between the ESWT and physical therapy groups. ESWT, extracorporeal shockwave therapy; SD, standard deviation; IV, inverse variance; $\mathrm{df}$, degrees of freedom.

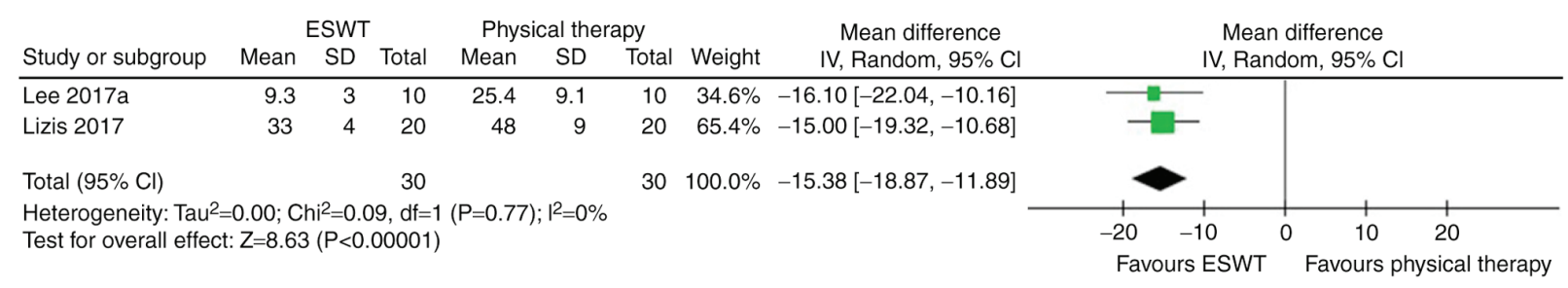

Figure 9. Forest plot for the Western Ontario and McMaster Universities osteoarthritis index compared between the ESWT and physical therapy groups. ESWT, extracorporeal shockwave therapy; SD, standard deviation; IV, inverse variance; df, degrees of freedom.

included in the present meta-analysis weakened the strength of the results regarding certain outcome measures such as ROM which only used 2 datasets, thereby necessitating the substantiation of the future results by a large number of RCTs.

In the ESWT group vs. physical therapy group analysis, the outcome indicators included the VAS, ROM and WOMAC scores. In terms of pain relief, the shockwave was significantly superior to physical exercise, albeit the heterogeneity in the two groups was slightly high $\left(\mathrm{P}=0.13, \mathrm{I}^{2}=56 \%\right)$. Thus, the reliability of the results was limited and hence, domestic studies from China were screened, revealing that the pain relief effect of ESWT was better than that of pure physical exercise therapy (3). Therefore, the small sample size may be the cause of heterogeneity. Furthermore, there is a similar meta-analysis (7), which produced similar results, with future clinical studies likely to confirm the results of the present study. Of note, in the ESWT group, the ROM $(18,23)$ and WOMAC $(22,23)$ score were significantly better than those in the physical therapy group and no obvious heterogeneity was detected in the two groups in the present study.

Of note, the present meta-analysis has several limitations. First, the number of relevant RCTs on the treatment of osteoarthritis was relatively low and the quality of the domestic studies was unreliable. Also, only studies published in English language were included, and hence, relevant studies published in other languages may have been missed. Furthermore, only seven studies were included and the sample size of each study was small, which limited the statistical power of the present meta-analysis. In addition, due to the limitation of the number of included studies, the therapeutic effects of the shockwaves of different energies were not analyzed, and all were combined into one shockwave group, which may have 
led to deviations of the results. Furthermore, the severity of OA prior to treatment may have also led to high heterogeneity and affected the reliability of the results. Finally, due to the limited literature, it was not possible to evaluate publication bias or to analyze functional outcomes e.g. post-operative quadriceps strength measures and the treatment costs due to insufficient data. Therefore, high-quality and large-scale clinical trials and systemic reviews are essential to confirm these results in the future.

In conclusion, in the treatment of KOA, the effects of ESWT are superior to those of placebo and physical therapy. Besides the pain relief, ESWT increases the mobility of the knee joint and may reduce the LI and WOMAC score. The evidence supported the selection of shock wave for the treatment of KOA patients; however, due to the lack of relevant high-quality literature and similar previous meta-analyses, and the fact that certain studies did not utilize a blinding method, the results require to be further substantiated.

\section{Acknowledgements}

Not applicable.

\section{Funding}

This study was supported by the Beijing Natural Science Foundation (grant no. 7182146), the Capital's Funds for Health Improvement and Research (grant no. CFH2018-4-40611), Beijing Municipal Science \& Technology Commission (grant no. Z181100001718058), the Fundamental Research Funds for the Central Universities (grant no. 3332018168), Graduate Innovation Foundation of Peking Union Medical College (grant no. 2017-1002-2-26) and the National Natural Science Foundation of China (grant nos. 81672236, 81802224 and 81871830).

\section{Availability of data and materials}

The datasets used and/or analyzed during the present study are available from the corresponding author on reasonable request.

\section{Authors' contributions}

WS and FG conceived and designed the study. TL, TZ and JM collected the data and performed the literature search. All authors were involved in writing the manuscript. All authors read and approved the final manuscript.

\section{Ethics approval and consent to participate}

Not applicable.

\section{Patient consent for publication}

Not applicable.

\section{Competing interests}

The authors declare that they have no competing interests.

\section{References}

1. Ji Q, Wang P and He C: Extracorporeal shockwave therapy as a novel and potential treatment for degenerative cartilage and bone disease: Osteoarthritis. A qualitative analysis of the literature. Prog Biophys Mol Biol 121: 255-265, 2016.

2. Hsu SL, Cheng JH, Wang CJ, Ko JY and Hsu CH: Extracorporeal shockwave therapy enhances expression of Pdia-3 which is a key factor of the 1 $\alpha, 25$-dihydroxyvitamin D 3 rapid membrane signaling pathway in treatment of early osteoarthritis of the knee. Int J Med Sci 14: 1220-1230, 2017.

3. Li JW, Zheng SJ, Zhang JC, Huang JJ and Liu XG: Effect of acupuncture plus different frequency shock-wave interventions on pain reactions and motor function in knee osteoarthritis patients. Zhen Ci Yan Jiu 40: 300-303, 2015 (In Chinese).

4. Pu W, Yujing Z, Xiaofei W, Xiaotian Y, Chengqi H and Chuan L: Effects of extracorporeal shock wave therapy on cartilage protection and subchondral bone remodeling in rabbits osteoarthritis induced by ACLT. Ann Phys Rehabil Med 57: e37, 2014

5. Maricar N, Callaghan MJ, Parkes MJ, Felson DT and O'Neill TW: Clinical assessment of effusion in knee osteoarthritis-A systematic review. Semin Arthritis Rheum 45: 556-563, 2016.

6. Kim JH, Kim JY, Choi CM, Lee JK, Kee HS, Jung KI and Yoon SR: The dose-related effects of extracorporeal shock wave therapy for knee osteoarthritis. Ann Rehabil Med 39: 616-623, 2015.

7. Liao CD, Tsauo JY, Liou TH, Chen HC and Huang SW: Clinical efficacy of extracorporeal shockwave therapy for knee osteoarthritis: A systematic review and meta-regression of randomized controlled trials. Clin Rehabil 8: 269215519846942, 2019.

8. Hou, X.D., H.B. Liu, and K.M. Liu, Effects of extracorporeal shock wave therapy on interleukin-1beta and matrix metalloproteinase-13 expression in rabbits with knee osteoarthritis. Zhong Guo Zu Zhi Gong Cheng Yan Jiu Za Zhi 18: 2397-2402, 2014 (In Chinese).

9. Zhao Z, Ji H, Jing R, Liu C, Wang M, Zhai L, Bai X and Xing G: Extracorporeal shock-wave therapy reduces progression of knee osteoarthritis in rabbits by reducing nitric oxide level and chondrocyte apoptosis. Arch Orthop Trauma Surg 132: 1547-1553, 2012

10. Yoon SR and Kim JH: Effect of extracorporeal shock wave therapy on knee osteoarthritis. Ann Phys Rehabil Med 57: e37-e38, 2014.

11. Wang P, Liu C, Yang XT, Wei XF, Zhou YJ, Yang L and He CQ: Effect of extracorporeal shock wave therapy on cartilage and subchondral bone remodeling in rabbits with ACLT-induced osteoarthritis. Sichuan Da Xue Xue Bao Yi Xue Ban 45: 120-125, 2014 (In Chinese).

12. Frisbie DD, Kawcak CE and McIlwraith CW: Evaluation of the effect of extracorporeal shock wave treatment on experimentally induced osteoarthritis in middle carpal joints of horses. Am J Vet Res 70: 449-454, 2009.

13. Ochiai N, Ohtori S, Sasho T, Nakagawa K, Takahashi K, Takahashi N, Murata R, Takahashi K, Moriya H, Wada Y and Saisu T: Extracorporeal shock wave therapy improves motor dysfunction and pain originating from knee osteoarthritis in rats. Osteoarthritis Cartilage 15: 1093-1096, 2007.

14. Mueller M, Bockstahler B, Skalicky M, Mlacnik E and Lorinson D: Effects of radial shockwave therapy on the limb function of dogs with hip osteoarthritis. Vet Rec 160: 762-765, 2007.

15. Imamura M, Alamino S, Hsing WT, Alfieri FM, Schmitz C and Battistella LR: Radial extracorporeal shock wave therapy for disabling pain due to severe primary knee osteoarthritis. J Rehabil Med 49: 54-62, 2017.

16. Wright JG, Swiontkowski MF and Tolo VT: Meta-analyses and systematic reviews: New guidelines for JBJS. J Bone Joint Surg Am 94: 1537, 2012.

17. Jokstad A: Cochrane collaboration systematic reviews may be based on trials not approved by a research ethics committee. Clin Exp Dent Res 3: 179-182, 2017.

18. Chen TW, Lin CW, Lee CL, Chen CH, Chen YJ, Lin TY and Huang MH: The efficacy of shock wave therapy in patients with knee osteoarthritis and popliteal cyamella. Kaohsiung J Med Sci 30: 362-370, 2014.

19. Cho SJ, Yang JR, Yang HS and Yang HE: Effects of extracorporeal shockwave therapy in chronic stroke patients with knee osteoarthritis: A pilot study. Ann Rehabil Med 40: 862-870, 2016. 
20. Elerian AE, Ewidea TMA and Ali N: Effect of shock wave therapy versus corticosteroid injection in management of knee osteoarthritis. Int J Physiother 3: 246-251, 2016.

21. Zhao Z, Jing R, Shi Z, Zhao B, Ai Q and Xing G: Efficacy of extracorporeal shockwave therapy for knee osteoarthritis: A randomized controlled trial. J Surg Res 185: 661-666, 2013.

22. Lee JH, Lee S, Choi S, Choi YH and Lee K: The effects of extracorporeal shock wave therapy on the pain and function of patients with degenerative knee arthritis. J Phys Ther Sci 29: 536-538, 2017
23. Lizis P, Kobza W and Manko G: Extracorporeal shockwave therapy vs. kinesiotherapy for osteoarthritis of the knee: A pilot randomized controlled trial. J Back Musculoskelet Rehabil 30: 1121-1128, 2017.

(c) (i) () $\odot$ This work is licensed under a Creative Commons Attribution-NonCommercial-NoDerivatives 4.0 International (CC BY-NC-ND 4.0) License. 